\title{
A Patient Portal-Based Commitment Device to Improve Adherence with Screening for Colorectal Cancer: a Retrospective Observational Study
}

\author{
Erin E. Hahn, $P h D, M P H^{1,2,3}(\mathbb{D})$, Aileen Baecker, $P h D^{7}$, Ernest Shen, $P h D^{7}$, \\ Eric C. Haupt, ScM ${ }^{7}$, Wahid Wakach, BIT', Andre Ahuja, MSc ${ }^{4}$, Tracy M. Imley, MD', \\ Michael K. Gould, MD, MS ${ }^{1,2}$, and Michael Kanter, MD
}

\begin{abstract}
'Department of Research and Evaluation, Kaiser Permanente Southern California, 100 S. Los Robles Ave, Pasadena, CA, USA; ${ }^{2}$ Kaiser Permanente Bernard J. Tyson School of Medicine, Department of Health Systems Science, Pasadena, CA, USA; ${ }^{3} J o n s s o n$ Comprehensive Cancer Center, University of California Los Angeles, Los Angeles, CA, USA; ${ }^{4}$ Southern California Permanente Medical Group, Pasadena, CA, USA; ${ }^{5}$ aiser Permanente Bernard J. Tyson School of Medicine, Department of Clinical Science, Pasadena, CA, USA.
\end{abstract}

BACKGROUND: Despite significant investment in colorectal cancer (CRC) screening, $40 \%$ of US adults are not up-to-date. Commitment devices, which are psychologically tailored approaches to enforce health goals, may be an effective method to increase CRC screening.

OBJECTIVE: Compare the effectiveness of a commitment device (patient self-ordering fecal immunochemical test (FIT) kits) to standard CRC screening outreach.

DESIGN: A retrospective observational study.

PARTICIPANTS: Participants were > 49 years and < 75 years, had no history of CRC, and were eligible for CRC screening.

INTERVENTION: An electronic screening reminder with an embedded order button allowed participants to order FIT kits directly from a patient portal. Those who used the order button were promptly sent a kit; those who did not were later mailed kits.

MAIN MEASURES: Primary outcome was completion of FIT kits. Secondary outcomes included number of days to completion, completion of follow-up for positive results, and CRC diagnosis; we also examined prior use of FIT kit. We used inverse probability of treatment weights to control for pretreatment imbalances.

KEY RESULTS: The cohort comprised 176,231 participants: 53\% female; median age was 59; $11 \%$ were Asian, 21\% Hispanic/Latino, 7\% black, 51\% White, 3\% other/ mixed race. Approximately $10 \%(N=16,918)$ used the button. Using inverse probability of treatment weights, we found that those who used the button had 3.8 times the odds of completing a kit compared to participants who did not (odds ratio, 3.77; 95\% confidence interval, 3.573.98). Within the button group, $63 \%$ of those eligible completed a FIT kit in the year prior to the button compared to $87 \%$ in the year after the button became available $(p<0.0001)$.

\section{Prior Presentation}

Study findings were presented as an oral abstract presentation at the Academy Health Annual Research Meeting, June 24-26, 2018, Seattle, WA, and at the Health Care Systems Research Network Conference, April 8-10, 2019, Portland, OR.

Received April 16, 2020

Accepted December 3, 2020

Published online January 20, 2021
CONCLUSION: The ability to self-order screening kits may act as a commitment device that increases CRC screening. Scalable tools leveraging existing patient portals such as this can complement existing CRC outreach strategies.

KEY WORDS: colorectal cancer screening; commitment device; patient portal; patient self-management.

$\mathrm{J}$ Gen Intern Med 36(4):952-60

DOI: $10.1007 / \mathrm{s} 11606-020-06392-y$

(C) Society of General Internal Medicine 2021

\section{INTRODUCTION}

\section{Background}

Colorectal cancer (CRC) screening is our most effective tool for reducing $\mathrm{CRC}$ incidence and mortality, with a grade A recommendation from the United States Preventive Service Task Force. ${ }^{1}$ CRC is the second leading cause of cancerrelated deaths in the USA, and mortality risk is significantly higher in unscreened individuals; thus, it is a national priority to increase CRC screening rates. ${ }^{2}$ The Centers for Disease Control have a goal of regular CRC screening in $80 \%$ of eligible patients over the age of $50 .{ }^{3}$ Unfortunately, approximately $40 \%$ of US adults are not up to date on CRC screening $^{4,5}$ despite the availability of several screening modalities including home fecal occult blood test (FOBT) and fecal immunochemistry (FIT) kits and multiple CRC screening outreach campaigns. A recent systematic review and metaanalysis compared the effectiveness of different strategies to increase CRC screening. ${ }^{6}$ FOBT and FIT outreach, patient navigation, and clinician-directed interventions all resulted in net CRC screening increases, with multicomponent interventions demonstrating the greatest increase. ${ }^{6}$ However, CRC outreach programs are often constrained by costs and resource limitations. ${ }^{7,8}$ Thus, there is a need to develop and test novel, scalable strategies that complement multi-component CRC screening outreach programs to increase CRC screening. 
Advances in health information technology, including growing use and sophistication of online patient portals, present new opportunities for participation in CRC screening. ${ }^{9}$ Patient engagement is associated with increased uptake of preventive care, ${ }^{10,11}$ and patient portals have been shown to facilitate improvements in medication adherence, patient selfmanagement, hospital readmissions, patient experience, and disease prevention. ${ }^{12-18}$ Novel patient-facing tools designed for online portals have potential to increase use of CRC screening as part of multi-component CRC screening outreach strategies.

A relatively unexplored area in CRC screening is the use of commitment devices. Originally from the field of behavioral economics, commitment devices were initially described as tying the individual to behavioral acts by behavior change. ${ }^{19}$ This definition has been expanded to include multiple approaches to enforce individual's voluntarily imposed health goals (e.g., health screenings, exercise, weight loss) until they have accomplished their goals. ${ }^{20}$ For example, arranging to exercise with friends is a commitment device that would help meet a voluntary fitness goal; not showing up leads to disappointment of friends (consequence of the behavior). ${ }^{20} \mathrm{Com}$ mitment devices can be conceptualized as a type of patient engagement strategy to help ensure health goals such as cancer screenings are met.

\section{Objectives}

In this study, we evaluated the impact of an interactive interface within a patient portal that allowed patients to directly order a FIT kit with the click of a button within an integrated healthcare system, Kaiser Permanente Southern California (KPSC). As for many healthcare systems, CRC screening is an important initiative within KPSC, which has a 10-year goal to reduce CRC mortality by $50 \%{ }^{21}$ Under the current KPSC regional outreach strategy, eligible KSPC members who fit the KPSC guidelines for CRC screening receive mailed FIT kits annually at no cost, as well as outreach including a letter from the primary care provider introducing the kit and urging the member to complete it, and auto-reminder calls, reminder postcards, and reminder secure email messaging if screening is not complete, with materials available in English and Spanish. Clinician-directed strategies to increase CRC screening rates include point-of-care reminders built into the EHR. This has resulted in screening rates of over $80 \%$ for KPSC. ${ }^{8}$ However, gaps in screening exist within KPSC. In this study, we hypothesized that participants who use the order button will have higher rates of screening completion compared to non-users, with the order button acting as a commitment device. We evaluated completion of the FIT kit, number of days to completion, completion of recommended follow-up for positive FIT kit results, and CRC diagnoses. We also evaluated measures of patient engagement to examine if engagement was associated with use of the button, including past use of the patient portal, missed appointments, and total utilization of outpatient care, as well as past FIT kit use.

\section{METHODS}

\section{Study Design and Participants}

We conducted a retrospective observational study among KPSC members eligible for CRC screening. Eligible patients were $>49$ years of age and $<75$ years old, had no history of $\mathrm{CRC}$, had not completed CRC screening using any modality within the past 12 months, and had not completed a colonoscopy within the past 10 years or sigmoidoscopy within the past 5 years. (Note that KPSC members are identified as eligible 23 months prior to their 50th birthday, hence the inclusion of $>$ 49 years.) Patients on hospice or palliative care were excluded. All materials were available in English and Spanish.

\section{Setting}

KPSC provides comprehensive care to over 4.6 million members with a long-standing electronic health record (EHR). The EHR features an online patient portal (kp. org) which provides patient access to appointments, results, and information about past visits. The patient portal also features the Online Personal Action Plan (oPAP). The oPAP was designed to enhance patient engagement, potentially improving efficacy of outreach efforts for prevention and other health services. The oPAP synthesizes information from the EHR to provide tailored information about recommended services including cancer screenings, immunizations, heart health, and other preventive care services. It features interactive content with links to enable patient actions. The oPAP has been shown to be an effective tool for closing care gaps, such as overdue HbA1c testing for diabetes management and overdue screenings. ${ }^{22}$

\section{Intervention}

In 2016, the oPAP team developed the interactive order button interface for the portal. A CRC screening reminder with the embedded button allows patients due for a FIT kit to order the kit directly from the patient portal. Email reminders for CRC screening with the embedded FIT kit order button were sent to eligible KPSC members due for their annual CRC screening. Those who used the order button were promptly sent a FIT kit and removed from the regional FIT kit mailing list. Those who did not use the button were mailed kits as part of the standard CRC screening outreach strategy. We included any eligible member who used the button to order a FIT kit up to October 2, 2017, in the button-user group; non-users were the comparison group. The index date was either the date the reminder was sent out or the date the button was first clicked, whichever came first. Participants were followed over time from initial invitation to completion of the FIT kit, other colorectal screening, terminated membership, death, or until May 1, 2018. All study activities were approved by the KPSC Institutional Review Board (IRB \#11624). 


\section{Variables}

Our primary outcome was completion of the FIT kit. Secondary outcomes included number of days to complete the kit and completion of recommended follow-up for positive results. We identified completion of the FIT kit using current procedural terminology (CPT) codes from the EHR and results of the test were designated as either "positive" or "negative," or were indeterminate. Time to completion was calculated using the result date. We also evaluated diagnosis of CRC during the study period. We identified newly diagnosed cancers using ICD-10 diagnosis codes (C18.0, C18.2-9, C19.X, C20.X) and confirmed with chart review. For those diagnosed with CRC during the study period, we extracted pathological cancer stage from pathology reports (American Joint Committee on Cancer 8th edition).

We identified covariates from the EHR including demographic information on age, gender, race/ethnicity, need for interpreter, and primary medical center. Preferred primary medical center was determined using utilization records within the prior year. Zip code was used to estimate preferred (closest) medical center for members with no healthcare utilization data. We also included a weighted Charlson Comorbidity Index (CCI) score. ${ }^{23}$ The CCI was calculated using utilization data from the EHR 1 year prior to the index date, and the weighted score was generated using age and diagnostic (ICD) data; scores were categorized as $0,1,2,3$, and $\geq 4$.

Lacking a survey-based measure of patient engagement, we relied on proxy measures of engagement including number of patient portal logins in the past year, no-show outpatient appointments in the past year, and overall outpatient healthcare utilization in the past year. These variables were selected based on association with patient engagement demonstrated in the scientific literature, including the association of higher engagement and increased use of health services ${ }^{24-26}$ and use of patient portals. ${ }^{27-29}$

\section{Statistical Analysis}

Initial binary comparisons of demographic and engagement characteristics were calculated with chi-square tests. To evaluate potential differences in completion of FIT kits between those who used the order button and those who did not, we used propensity score methods to control for pretreatment imbalances commonly seen in observational studies. ${ }^{30,31}$ A logistic regression was run with the inverse probability of treatment weights to balance the study groups with respect to demographic and utilization characteristics. ${ }^{32}$ Odds ratios with $95 \%$ confidence intervals were computed to compare the odds of completing a FIT kit among those who used the button compared to eligible members who had active logins on the patient portal but did not use the button. Additionally, to evaluate the potential effect of engagement, we compared the odds of completing a FIT kit among those who logged in to the patient portal but did not use the button to those who had zero logins during the study period. Missing values were included in an "unknown" category, ensuring that propensity score weighting could be conducted on all observations. Propensity scores were computed using the R package Toolkit for Weighting and Analysis of Nonequivalent Groups (TWANG), using age, gender, race and ethnicity, need for a language interpreter, preferred medical center, number of patient portal logins in the past year, no-show outpatient appointments in the past year, overall outpatient healthcare utilization in the past year, and the CCI. The

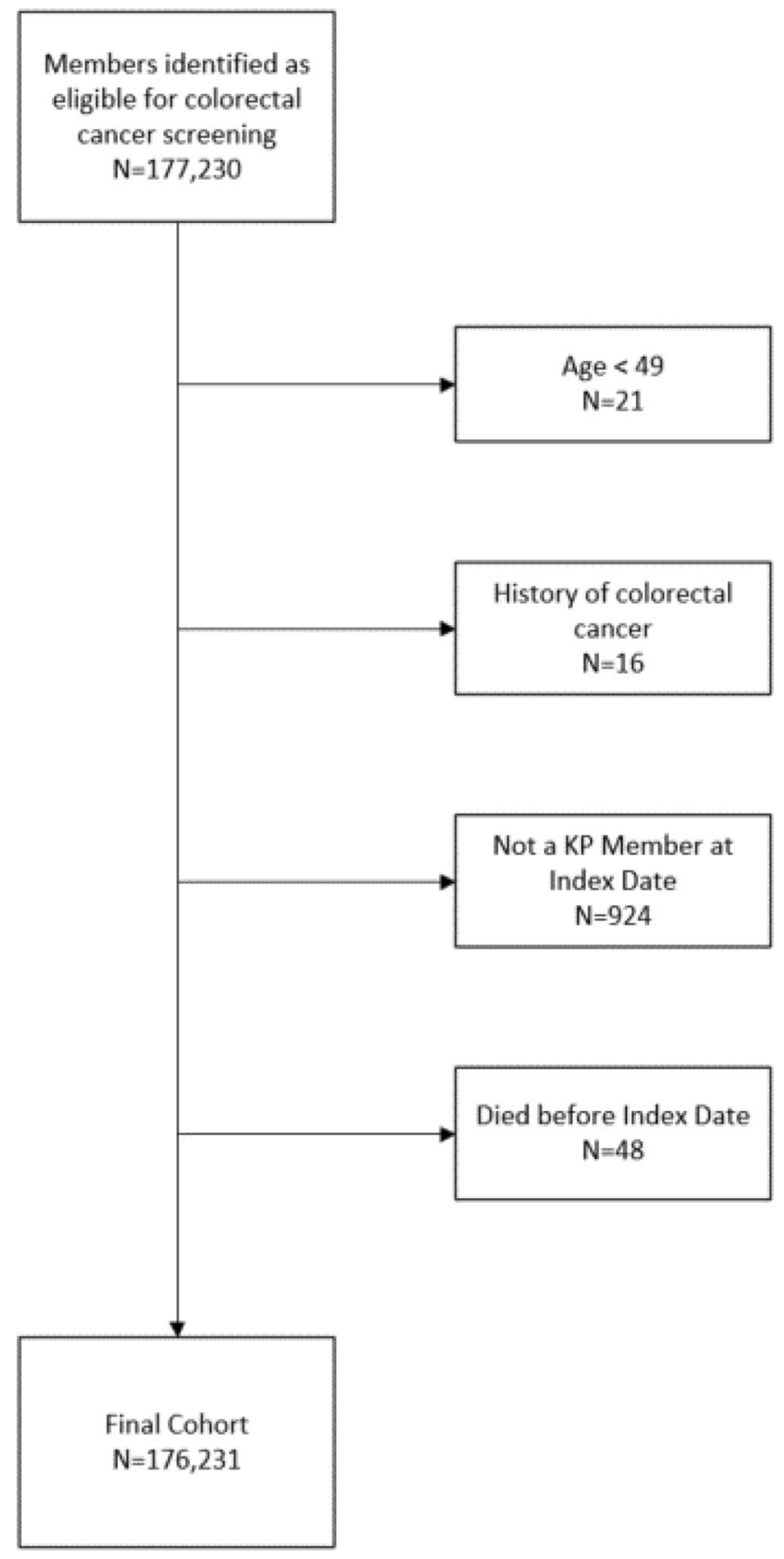

Fig. 1 Cohort diagram, Kaiser Permanente Southern California members eligible for annual fecal immunochemistry testing kits for colorectal cancer screening, $N=\mathbf{1 7 6 , 2 3 1}$. 
Table 1 Patient Demographics and Engagement Characteristics of Kaiser Permanente Southern California Members Eligible for Home Colorectal Cancer Screening and Received the Self-Order Button Message on the Patient Portal Between December 14, 2016, and July 19, 2017, Unweighted Data, $N=176,231$

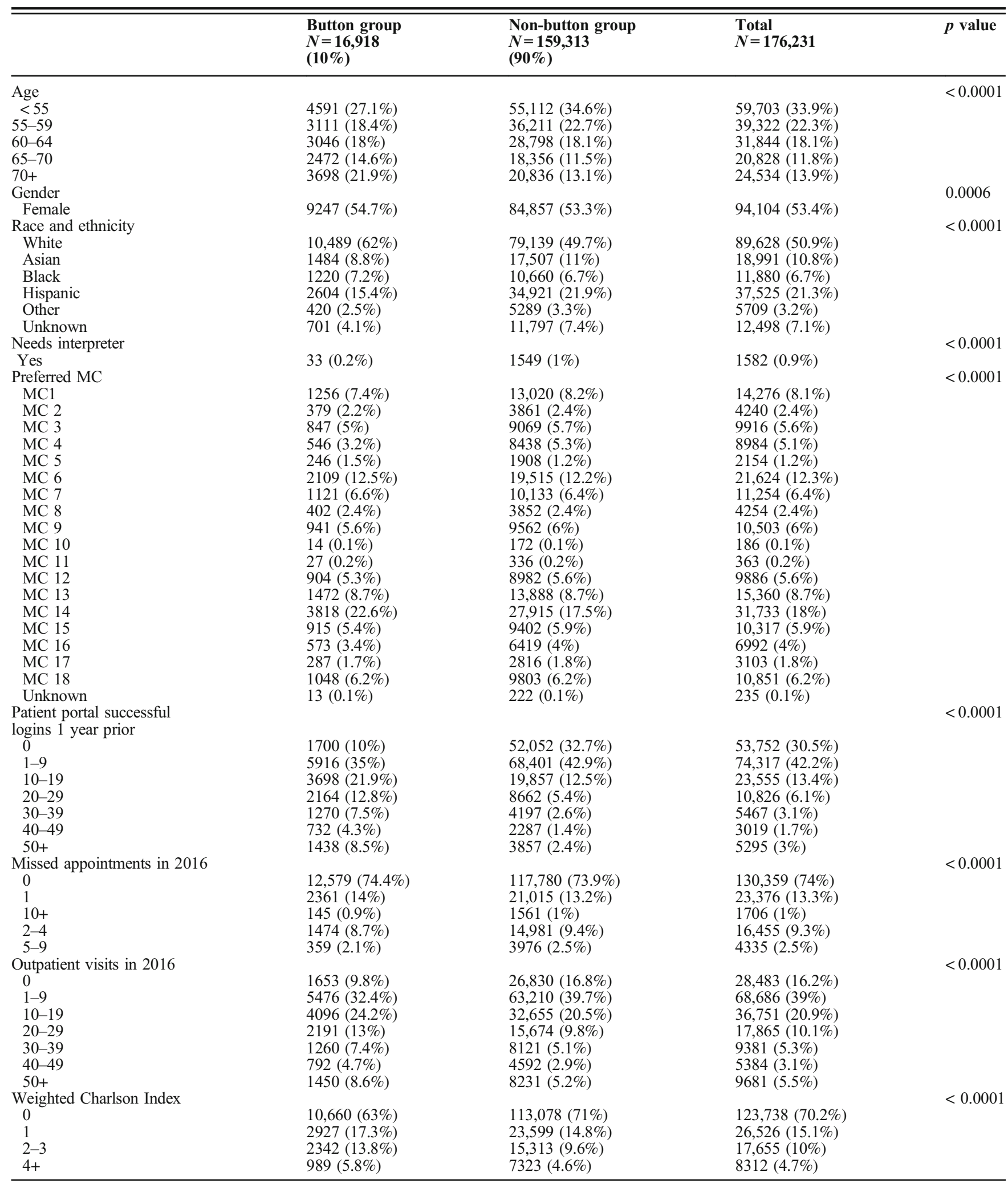

MC medical center 
average treatment effect (ATE) was estimated using KS Max as a stopping method.

To determine if the button led to patients completing CRC screening who had not been up-to-date on screening in the past, we compared the number of those in the button group who completed the FIT kit within the prior year to those in the button group who completed a FIT kit after the button became available using McNemar's test. Analyses were conducted in SAS, version 9.4, and $\mathrm{R}$, version 3.2.2.

\section{RESULTS}

\section{Participants}

The cohort consisted of 176,231 participants (Fig. 1); we excluded those erroneously identified including 21 who were $<49$ years, 16 who had a history of colorectal cancer, 924 participants who were not members at the index date, and 48 who died before the index date. Overall, 53\% of the cohort were female; the median age was 59 , with $34 \%$ aged $49-55$, $22 \%$ aged $56-59,18 \%$ aged $64,12 \%$ aged $65-70$, and $14 \%$ aged 71 or older (Table 1); 11\% were Asian, 21\% Hispanic/ Latino, $7 \%$ black, $51 \%$ White, $3 \%$ other/mixed race, and $7 \%$ unknown. Over the course of the study period, approximately $4 \%$ terminated KP membership $(N=6295)$ and $<1 \%$ died $(N=669)$.

\section{Descriptive Data}

Approximately $10 \%(N=16,918)$ used the oPAP button to request a FIT kit. In bivariate comparisons, we found significant differences between the button and nonbutton groups on both demographics and proxy measures of engagement. A significantly higher proportion of the button group were white race $(62 \%$ in the button group vs. $50 \%$ non-button), and were over the age of 71 ( $22 \%$ vs. $13 \%$ ); a significantly smaller proportion of the button group were aged 49-55 (27\% vs. 35\%), were Asian (9\% vs. $11 \%)$ or Hispanic (15\% vs. $22 \%)$, or required an interpreter ( $2 \%$ vs. $4 \%$ ). For proxy measures of engagement, the button group had a significantly higher proportion of $\geq 20$ patient portal logins in the past year (33\% vs. 12\%) as well as a significantly higher proportion of frequent outpatient utilization, defined as 20 or more outpatient visits in the past year (34\% vs. 23\%). Those in the button group had a significantly higher proportion of those with a CCI score of 2 or $3(14 \%$ vs. $10 \%)$ and $\geq 4(6 \%$ vs. $4 \%)$.

\section{Primary Outcome}

Among those in the button group, $83 \%$ completed a FIT kit, compared to $37 \%$ of the non-button group (Fig. 2). Using the inverse probability of treatment weights to balance the groups

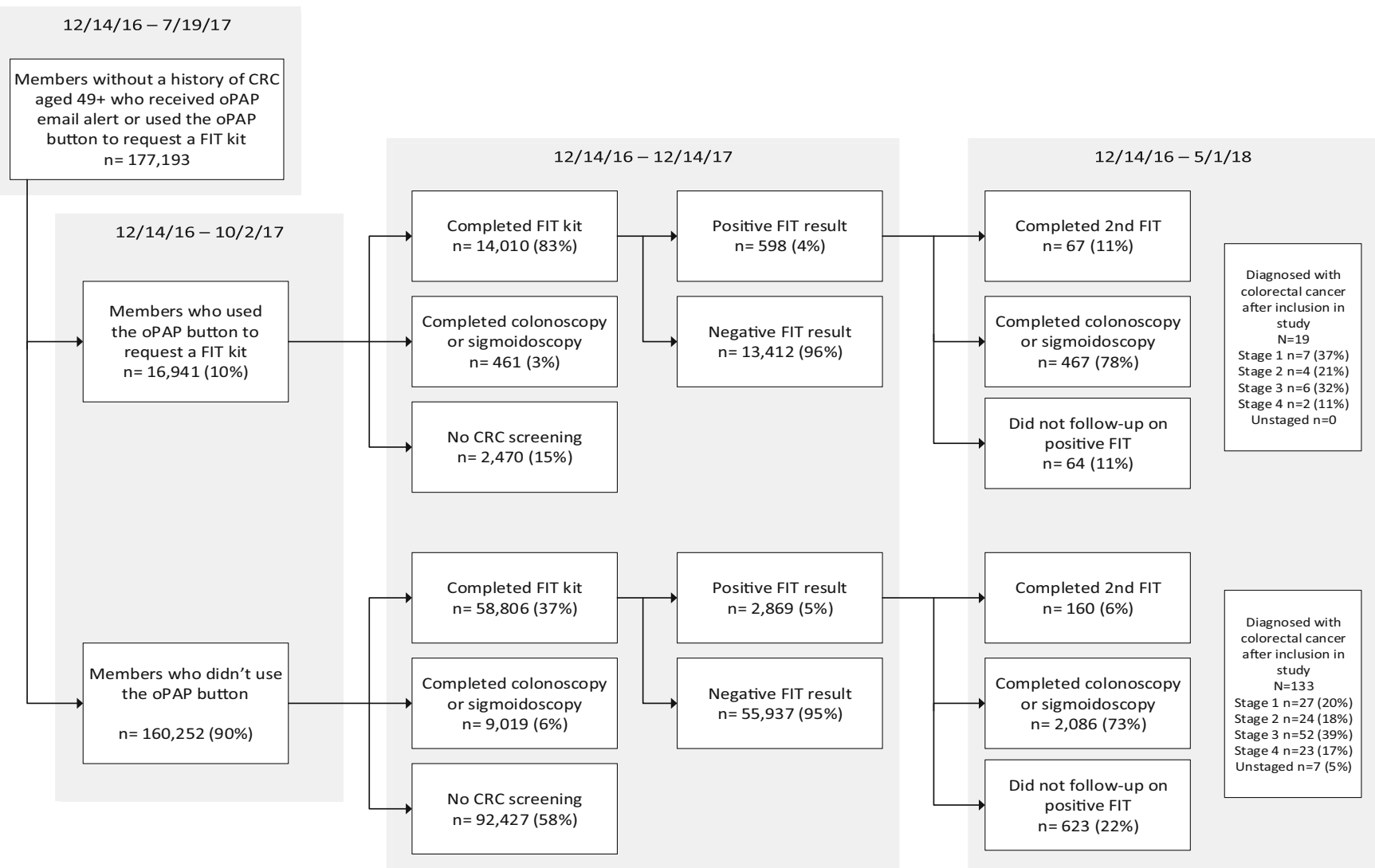

Fig. 2 Flow diagram of button and non-button group completion of initial and follow-up colorectal cancer screening, $N=176,231$. 
Table 2 Weighted Patient Demographics and Engagement Characteristics of Kaiser Permanente Southern California Members Eligible for Home Colorectal Cancer Screening and Received the Self-Order Button Message on the Patient Portal Between December 14, 2016, and July 19, 2017, $N=176,231$

\begin{tabular}{|c|c|c|c|c|}
\hline & $\begin{array}{l}\text { Logged in, no button } \\
N=98,306(62 \%)\end{array}$ & $\begin{array}{l}\text { Did not log in, no button } \\
N=61,007(38 \%)\end{array}$ & $\begin{array}{l}\text { Button group } \\
N=16,918(11 \%)\end{array}$ & $\begin{array}{l}\text { Rao-Scott chi-square } \\
p \text { value }\end{array}$ \\
\hline \multicolumn{4}{|l|}{ Age } & \multirow[t]{6}{*}{0.6557} \\
\hline$<55$ & $59,801(34 \%)$ & $59,738(34.4 \%)$ & $57,971(34.1 \%)$ & \\
\hline $55-59$ & $39,149(22.3 \%)$ & $38,945(22.4 \%)$ & $37,153(21.8 \%)$ & \\
\hline $60-64$ & $31,635(18 \%)$ & $31,436(18.1 \%)$ & $31,294(18.4 \%)$ & \\
\hline $65-70$ & $20,699(11.8 \%)$ & $20,133(11.6 \%)$ & $20,261(11.9 \%)$ & \\
\hline $70+$ & $24,360(13.9 \%)$ & $23,284(13.4 \%)$ & $23,523(13.8 \%)$ & \\
\hline \multicolumn{4}{|l|}{ Gender } & \multirow[t]{3}{*}{0.2102} \\
\hline Female & $93,777(53.4 \%)$ & $92,476(53.3 \%)$ & $89,502(52.6 \%)$ & \\
\hline Male & $81,867(46.6 \%)$ & $81,060(46.7 \%)$ & $80,700(47.4 \%)$ & \\
\hline \multicolumn{4}{|l|}{ Race and ethnicity } & \multirow[t]{7}{*}{0.1077} \\
\hline White & $89,536(51 \%)$ & $88,060(50.7 \%)$ & $89,014(52.3 \%)$ & \\
\hline Asian & $18,882(10.8 \%)$ & $18,719(10.8 \%)$ & $18,602(10.9 \%)$ & \\
\hline Black & $11,834(6.7 \%)$ & $11,622(6.7 \%)$ & $11,173(6.6 \%)$ & \\
\hline Hispanic & $37,330(21.3 \%)$ & $37,181(21.4 \%)$ & $34,257(20.1 \%)$ & \\
\hline Other & $5642(3.2 \%)$ & $5496(3.2 \%)$ & $5414(3.2 \%)$ & \\
\hline Unknown & $12,420(7.1 \%)$ & $12,457(7.2 \%)$ & $11,742(6.9 \%)$ & \\
\hline \multicolumn{4}{|l|}{ Needs interpreter } & \multirow{4}{*}{0.0228} \\
\hline Yes & $6589(3.8 \%)$ & $6706(3.9 \%)$ & $5444(3.2 \%)$ & \\
\hline No & $167,496(95.4 \%)$ & $165,243(95.2 \%)$ & $163,567(96.1 \%)$ & \\
\hline Unknown & $1559(0.9 \%)$ & $1587(0.9 \%)$ & $1191(0.7 \%)$ & \\
\hline \multicolumn{4}{|l|}{ Preferred medical center } & \multirow[t]{20}{*}{0.9972} \\
\hline MC 1 & $14,278(8.1 \%)$ & $14,094(8.1 \%)$ & $13,864(8.1 \%)$ & \\
\hline MC 2 & $4307(2.5 \%)$ & $4146(2.4 \%)$ & $4237(2.5 \%)$ & \\
\hline MC 3 & $9894(5.6 \%)$ & $9813(5.7 \%)$ & $9535(5.6 \%)$ & \\
\hline MC 4 & $8814(5 \%)$ & $8875(5.1 \%)$ & $7906(4.6 \%)$ & \\
\hline MC 5 & $2179(1.2 \%)$ & $1970(1.1 \%)$ & $2238(1.3 \%)$ & \\
\hline MC 6 & $21,543(12.3 \%)$ & $21,013(12.1 \%)$ & $20,676(12.1 \%)$ & \\
\hline MC 7 & $11,253(6.4 \%)$ & $11,212(6.5 \%)$ & $11,107(6.5 \%)$ & \\
\hline MC 8 & $4209(2.4 \%)$ & $4291(2.5 \%)$ & $3892(2.3 \%)$ & \\
\hline MC 9 & $10,522(6 \%)$ & $10,439(6 \%)$ & $10,313(6.1 \%)$ & \\
\hline MC 10 & $176(0.1 \%)$ & $168(0.1 \%)$ & $144(0.1 \%)$ & \\
\hline MC 11 & $357(0.2 \%)$ & $344(0.2 \%)$ & $377(0.2 \%)$ & \\
\hline MC 12 & $9871(5.6 \%)$ & $9759(5.6 \%)$ & $9388(5.5 \%)$ & \\
\hline MC 13 & $15,390(8.8 \%)$ & $15,333(8.8 \%)$ & $15,167(8.9 \%)$ & \\
\hline MC 14 & $31,642(18 \%)$ & $31,195(18 \%)$ & $30,629(18 \%)$ & \\
\hline MC 15 & $10,164(5.8 \%)$ & $10,051(5.8 \%)$ & $9983(5.9 \%)$ & \\
\hline MC 16 & $6870(3.9 \%)$ & $6855(4 \%)$ & $6642(3.9 \%)$ & \\
\hline MC 17 & $3097(1.8 \%)$ & $3150(1.8 \%)$ & $3042(1.8 \%)$ & \\
\hline MC 18 & $10,855(6.2 \%)$ & $10,609(6.1 \%)$ & $10,933(6.4 \%)$ & \\
\hline Unknown & $222(0.1 \%)$ & $221(0.1 \%)$ & $129(0.1 \%)$ & \\
\hline \multicolumn{4}{|l|}{ KP.org successful logins 1 year prior } & \multirow[t]{8}{*}{0.0974} \\
\hline 0 & $53,413(30.4 \%)$ & $53,586(30.9 \%)$ & $49,954(29.3 \%)$ & \\
\hline $1-9$ & $74,171(42.2 \%)$ & $73,889(42.6 \%)$ & $72,843(42.8 \%)$ & \\
\hline $10-19$ & $23,509(13.4 \%)$ & $23,160(13.3 \%)$ & $23,239(13.7 \%)$ & \\
\hline $20-29$ & $10,829(6.2 \%)$ & $10,429(6 \%)$ & $10,700(6.3 \%)$ & \\
\hline $30-39$ & $5440(3.1 \%)$ & $5182(3 \%)$ & $5308(3.1 \%)$ & \\
\hline $40-49$ & $3009(1.7 \%)$ & $2716(1.6 \%)$ & $2957(1.7 \%)$ & \\
\hline $50+$ & $5272(3 \%)$ & $4573(2.6 \%)$ & $5200(3.1 \%)$ & \\
\hline \multicolumn{4}{|l|}{ Missed appointments in 2016} & 0.1059 \\
\hline 0 & $129,973(74 \%)$ & $127,898(73.7 \%)$ & $127,845(75.1 \%)$ & \\
\hline 1 & $23,315(13.3 \%)$ & $23,144(13.3 \%)$ & $22,038(12.9 \%)$ & \\
\hline $10+$ & $1691(1 \%)$ & $1717(1 \%)$ & $1430(0.8 \%)$ & \\
\hline $2-4$ & $16,411(9.3 \%)$ & $16,457(9.5 \%)$ & $15,131(8.9 \%)$ & \\
\hline $5-9$ & $4253(2.4 \%)$ & $4320(2.5 \%)$ & $3758(2.2 \%)$ & \\
\hline Outpatient visits in 2016 & & & & 0.8898 \\
\hline 0 & $28,358(16.1 \%)$ & $28,182(16.2 \%)$ & $27,949(16.4 \%)$ & \\
\hline $1-9$ & $68,563(39 \%)$ & $68,388(39.4 \%)$ & $65,874(38.7 \%)$ & \\
\hline $10-19$ & $36,588(20.8 \%)$ & $36,460(21 \%)$ & $35,888(21.1 \%)$ & \\
\hline $20-29$ & $17,785(10.1 \%)$ & $17,347(10 \%)$ & $16,876(9.9 \%)$ & \\
\hline $30-39$ & $9396(5.3 \%)$ & $9063(5.2 \%)$ & $9203(5.4 \%)$ & \\
\hline $40-49$ & $5342(3 \%)$ & $4925(2.8 \%)$ & $5323(3.1 \%)$ & \\
\hline $50+$ & $9612(5.5 \%)$ & $9171(5.3 \%)$ & $9088(5.3 \%)$ & \\
\hline Weighted Charlson Index & & & & 0.5008 \\
\hline 0 & $123,261(70.2 \%)$ & $123,200(71 \%)$ & $120,470(70.8 \%)$ & \\
\hline 1 & $26,515(15.1 \%)$ & $26,022(15 \%)$ & $25,536(15 \%)$ & \\
\hline $2-3$ & $17,618(10 \%)$ & $16,761(9.7 \%)$ & $16,390(9.6 \%)$ & \\
\hline $4+$ & $8249(4.7 \%)$ & $7553(4.4 \%)$ & $7806(4.6 \%)$ & \\
\hline
\end{tabular}

MC medical center 


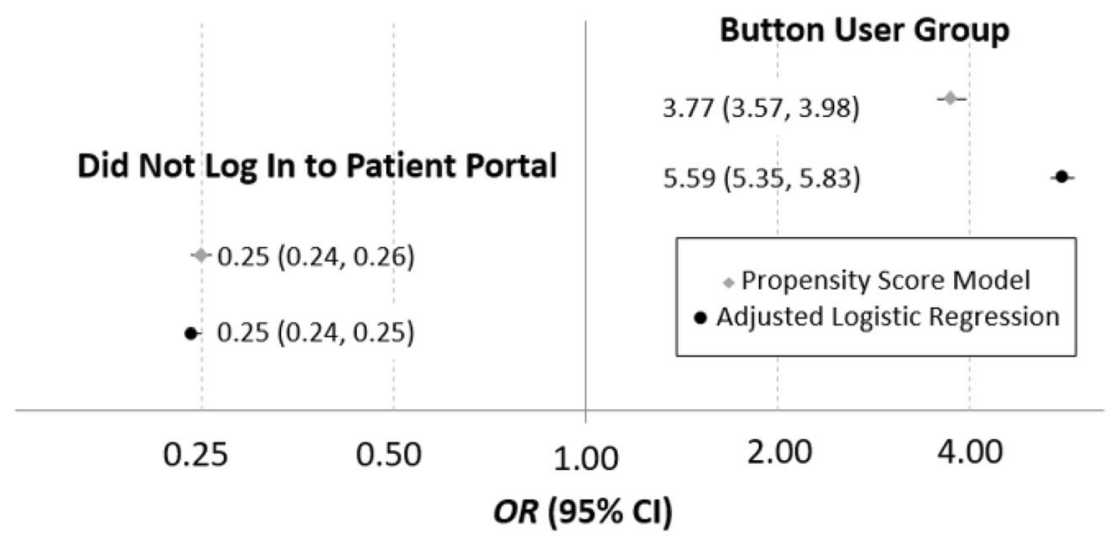

Propensity Score Model Variables: gender, race/ethnicity, age category, interpreter needed, most recently utilized medical center, prior patient portal logins, prior missed appointments, prior utilization, weighted Charlson score

Fig. 3 Odds ratios comparing the button-user group to those who logged in to the patient portal but did not click the button.

on demographic and proxy engagement characteristics (see weighted characteristics in Table 2), we found that those who used the FIT kit button had 3.8 times the odds of completing a FIT kit in comparison to participants who logged into the patient portal during the study period but did not use the button (odds ratio (OR), 3.77; 95\% confidence interval (CI), 3.573.98) adjusting for gender, race/ethnicity, age, interpreter needs, recently utilized medical center, prior patient portal logins, prior missed appointments, prior total utilization, and weighted CCI (Fig. 3). In the non-button group, those who did not login to the patient portal during the study period had $75 \%$ less odds of completing a FIT kit compared to those who logged in but not use the FIT kit button.

\section{Secondary Outcomes}

To estimate the impact of the button for patients who had not completed CRC screening in the past, we examined past FIT kit use in the button group. We determined that 12,081 (71\%) of the button-user group were KPSC members and eligible for a FIT kit in the year prior to the release of the button (Table 3). Of those, 63\% (7593) completed a FIT kit prior to the availability of the button compared to $87 \%(10,469)$ in the year after the button became available (McNemar's test $p<0.0001)$, an increase of 2876 patients.

The mean number of days to FIT kit completion was 56 days in the button group compared to 90 days in the non-button group $(p<0.001)$. Overall, $4 \%$ of the both groups who completed a FIT kit required additional CRC screening for positive or inconclusive results. For those patients who had a positive FIT result requiring additional screening, $8 \%$ of the button group did not have subsequent recommended screening vs. $18 \%$ in the non-button group.

We also examined CRC diagnosis: 19 patients $(\leq 1 \%)$ in the button group were diagnosed with a new primary CRC during the study period: $37 \%$ stage I, $21 \%$ stage $2,32 \%$ stage III, and $11 \%$ stage IV. Among the non-button group, $133(\leq 1 \%)$ had a new primary CRC diagnosed during the study period: $20 \%$ stage I, $18 \%$ stage 2, 39\% stage III, $17 \%$ stage IV, and 5\% unstaged.

\section{DISCUSSION}

\section{Key Results}

In this observational study, we found that a significantly greater proportion of patients who self-ordered FIT kits completed the kit compared to those who received a FIT kit as part of a mailed outreach strategy ( $83 \%$ vs. $37 \%$ ). Using inverse probability of treatment weights to control for imbalances between the groups including demographics and proxy measures of patient engagement, we found that button users had 3.8 times the odds of completing the kit compared to the nonbutton group ( $\mathrm{OR}=3.77,95 \%$ CI 3.57-3.98). Importantly, in the button group, we found that only $63 \%$ of those eligible for

Table 3 Completion of FIT Kits for Participants in the Button-User Group Who Were Eligible for a FIT Kit 1 Year Prior in Prior Year Versus Post-Button Introduction $(n=12,081)$

\begin{tabular}{lllll}
\hline \hline & & Post-button FIT & & Yes \\
\cline { 3 - 5 } & & No & Total \\
\hline One year prior FIT & No & 1065 & 3423 & 4046 \\
& Yes & 547 & 7046 & 7593 \\
& Total & 1612 & 10,469 & 12,081 \\
\hline
\end{tabular}

McNemar's test $p<0.0001$ 
a FIT kit in the prior year completed a kit compared to $87 \%$ who completed a kit after the order button became available, an increase of $24 \%$ or 2876 patients. This demonstrates a potential impact of the button beyond those patients who were already engaged in CRC screening.

\section{Strengths and Limitations}

Our study has several strengths. Our large, racially/ ethnically diverse cohort is a significant strength, as well as the longitudinal follow-up data on FIT kit completion, abnormal FIT results, and diagnoses of CRC. Additionally, we were able to capture multiple measures of patient engagement including outpatient utilization, missed appointments, and patient portal logins in the prior year. Our study also has limitations, the most important the observational design. Participants were not randomized to receive the button message; all participants received the message and self-selected to use the button or not. The button group differed significantly from the non-button group; there was a higher proportion of white race in the button group, as well as higher proportion of portal log-ins and higher healthcare utilization, and fewer missed appointments. We used appropriate statistical methodology for causal inference to balance the groups with available covariates, including important measures of engagement, but omitted variable bias is a possibility and may limit generalizability of results. However, our results are similar to a recent RCT of patient self-order of CRC screening. ${ }^{33}$ Additionally, KPSC is an integrated system, and the structure of the system may be associated with screening results and not generalizable to other systems. KPSC has high baseline rates of screening and participants were likely exposed to multiple outreach strategies. This exposure may have "primed the pump" for use of the button and completion of screening; results in other settings may be different. The generalizability of the study findings might also depend on the availability and type of patient portal; generating accurate lists of eligible patients requires resources. However, as health systems and other delivery settings continue to invest in patient-facing health technology, the ability to create opportunities such as self-ordering CRC screening will increase.

\section{Interpretations}

Our findings are in line with a recent randomized controlled trial that examined the impact of patient selfordering CRC screening using an iPad, with the ability to order either a home screening kit or a colonoscopy. ${ }^{33}$ Participants who were randomized to the self-order arm had over twice the odds of completing screening than controls $(\mathrm{OR}=2.5$, CI 1.6-4.0). Based on these results, it is possible that the self-order button can act as an effective commitment device. ${ }^{34}$ This may be the case even for patients who were previously not engaged in CRC screening behaviors as our findings suggest. Although effects may be modest, patients using commitment devices can be more successful at achieving health goals including smoking cessation ${ }^{35}$ and physical activity/nutrition goals $^{36}$; even modest increases in CRC screening rates can have an impact on CRC diagnoses and outcomes. Only $10 \%$ used the button, which is also in line with recent systematic reviews of portals which found that $10-30 \%$ of patients used a portal function. ${ }^{37,38}$

This work demonstrates that patient self-ordering of CRC screening may help increase CRC screening rates. Scalable health IT tools leveraging existing patient portals such as this can serve as effective complements to existing CRC screening outreach strategies.

Corresponding Author: Erin E. Hahn, PhD, MPH; Department of Research and Evaluation, Kaiser Permanente Southern California, 100 S. Los Robles Ave, Pasadena, CA 91101, USA (e-mail: Erin.E. Hahn@kp.org).

Funding This study was supported by the Kaiser Permanente Southern California Care Improvement Research Team.

\section{Compliance with Ethical Standards:}

Conflict of Interest: The authors declare that they do not have a conflict of interest.

Disclaimer: The funding source had no role in the design and conduct of the study; collection, management, analysis, and interpretation of the data; preparation, review, or approval of the manuscript; and decision to submit the manuscript for publication.

\section{REFERENCES}

1. United States Preventive Services Task Force (USPSTF): Screening for Colorectal Cancer: US Preventive Services Task Force Recommendation Statement. Jama. 2016;315(23):2564-2575.

2. Wolf AM, Fontham ET, Church TR, et al. Colorectal cancer screening for average-risk adults: 2018 guideline update from the American Cancer Society. CA Cancer J Clin. 2018;68(4):250-281.

3. Centers for Disease Control (CDC): What CDC is doing about colorectal cancer. https://www.cdc.gov/cancer/colorectal/what_cdc_is_doing/index.htm. Accessed 15 Aug 2019

4. Green BB. Colorectal cancer control: Where have we been and where should we go next? JAMA Intern Med. 2018;178(12):1658-60.

5. de Moor JS, Cohen RA, Shapiro JA, et al. Colorectal cancer screening in the United States: trends from 2008 to 2015 and variation by health insurance coverage. J Prev Med. 2018;112:199-206.

6. Dougherty MK, Brenner AT, Crockett SD, Gupta S, Wheeler SB Coker-Schwimmer M, Cubillos L, Malo T, Reuland DS. Evaluation of interventions intended to increase colorectal cancer screening rates in the United States: a systematic review and meta-analysis. JAMA internal medicine. 2018;178(12):1645-58.

7. Klabunde C, Blom J, Bulliard JL, et al. Participation rates for organized colorectal cancer screening programmes: an international comparison. J Med Screen. 2015;22(3):119-126.

8. Levin TR, Corley DA, Jensen CD, Schottinger JE, Guinn VP, Zauber AG, Lee JK, Zhao WK, Udaltsova N, Ghai NR, Lee AT. Effects of organized colorectal cancer screening on cancer incidence and mortality in a large community-based population. Gastroenterology. 2018;155(5):1383-91 
9. Ricciardi L, Mostashari F, Murphy J, Daniel JG, Siminerio. A national action plan to support consumer engagement via e-health. Health Affairs 2013;32(2):376-384.

10. Green BB, Wang C-Y, Anderson ML, et al. An automated intervention with stepped increases in support to increase uptake of colorectal cancer screening: a randomized trial. Ann Intern Med. 2013;158(5 0 1):301.

11. Hibbard JH, Greene J, Overto V. Patients With Lower Activation Associated With Higher Costs; Delivery Systems Should Know Their Patients' 'Scores'. Health Affairs. 2013;32(2):216-222.

12. Kruse CS, Bolton K, Freriks G. The effect of patient portals on quality outcomes and its implications to meaningful use: a systematic review. J Med Internet Res. 2015;17(2):e44-e44.

13. Hamine S, Gerth-Guyette E, Faulx D, Green BB, Ginsburg AS. Impact of mHealth chronic disease management on treatment adherence and patient outcomes: a systematic review. J Med Internet Res. 2015; 17(2):e52-e52.

14. Dendere R, Slade C, Burton-Jones A, Sullivan C. Staib A, Janda M. Patient Portals Facilitating Engagement With Inpatient Electronic Medical Records: A Systematic Review. JMIR Publ: Adv Digit Open Sci. 2019;21(4):e12779.

15. Martínez Nicolás I, Lê Cook B, Flores M, et al. The impact of a comprehensive electronic patient portal on the health service use: an interrupted time-series analysis. Eur J Public Health. 2018;29(3):413418.

16. Mohammed MA, Montague J, Faisal M, Lamming $\mathbf{L}$. The value of a Patient Access Portal in primary care: a cross-sectional survey of 62,486 registered users in the UK. Universal Access in the Information Society. 2019

17. Grossman LV, Masterson Creber RM, Benda NC, Wright D, Vawdrey DK, Ancker JS. Interventions to increase patient portal use in vulnerable populations: a systematic review. J Am Med Inform Assoc. 2019;26(89):855-870

18. Vreugdenhil MMT, Ranke S, de Man Y, Haan MM, Kool RB. Patient and Health Care Provider Experiences With a Recently Introduced Patient Portal in an Academic Hospital in the Netherlands: Mixed Methods Study. JMIR Publ Adv Digit Open Sci. 2019;21(8): 13743.

19. Kiesler CA. Sakumura J. A test of a model for commitment. J Pers Soc Psychol. 1966;3(3):349.

20. Rogers T, Milkman KL, Volpp KG. Commitment Devices: Using Initiatives to Change Behavior. JAMA. 2014;311(20):2065-2066.

21. Schottinger JE, Kanter MH, Litman KC, et al. Using literature review and structured hybrid electronic/manual mortality review to identify system-level improvement opportunities to reduce colorectal cancer mortality. Jt Comm J Qual Patient Saf 2016;42(7):303-AP302.

22. Henry SL, Shen E, Ahuja A, Gould MK, Kanter MH. The Online Personal Action Plan: A Tool to Transform Patient-Enabled Preventive and Chronic Care. Am J Prev Med. 2016;51(1):71-77.

23. Charlson ME, Pompei P, Ales KL, Mackenzie CR. A new method of classifying prognostic comorbidity in longitudinal studies: development and validation. J Chronic Dis. 1987;40(5):373-383.

24. Shade SB, Steward WT, Koester KA, Chakravarty D, Myers JJ. Health information technology interventions enhance care completion, engagement in HIV care and treatment, and viral suppression among HIV- infected patients in publicly funded settings. J Am Med Inform Assoc. 2014;22(e1):e104-e111.

25. Kinney RL, Lemon SC, Person SD, Pagoto SL, Saczynski JS. The association between patient activation and medication adherence, hospitalization, and emergency room utilization in patients with chronic illnesses: a systematic review. Patient Educ Couns. 2015;98(5):545-552.

26. Remmers C, Hibbard J, Mosen DM, Wagenfield M, Hoye RE, Jones C. Is Patient Activation Associated With Future Health Outcomes and Healthcare Utilization Among Patients With Diabetes? J Ambul Care Manage. 2009;32(4):320-327.

27. Irizarry T, DeVito Dabbs A, Curran CR. Patient Portals and Patient Engagement: A State of the Science Review. JMIR Publ: Adv Digit Open Sci. 2015; 17(6):e148.

28. Pillemer F, Price RA, Paone S, et al. Direct Release of Test Results to Patients Increases Patient Engagement and Utilization of Care. PLoS One. 2016;11(6):e0154743.

29. Zhong $\mathbf{X}$, Liang $\mathbf{M}$, Sanchez $\mathbf{R}$, et al. On the effect of electronic patient portal on primary care utilization and appointment adherence. BMC Med Inform Decis Mak. 2018;18(1):84.

30. Rosenbaum PR, Rubin DB. The central role of the propensity score in observational studies for causal effects. Biometrika. 1983;70(1):41-55.

31. Austin PC. An introduction to propensity score methods for reducing the effects of confounding in observational studies. Multivar Behav Res. 2011;46(3):399-424

32. McCaffrey DF, Griffin BA, Almirall D, Slaughter ME, Ramchand $\mathbf{R}$, Burgette LF. A tutorial on propensity score estimation for multiple treatments using generalized boosted models. Stat Med. 2013;32(19):3388-3414.

33. Miller DP, Jr, Denizard-Thompson N, Weaver KE, et al. Effect of a digital health intervention on receipt of colorectal cancer screening in vulnerable patients: A randomized controlled trial. Ann Intern Med. 2018;168(8):550-557.

34. Mehta SJ, Asch DA. Does using an ipad make an intervention innovative? Ann Intern Med. 2018;168(8):592-593.

35. Giné X, Karlan D, Zinman J. Put Your Money Where Your Butt Is: A Commitment Contract for Smoking Cessation. Am Econ J: Appl Econ. 2010;2(4):213-235.

36. Milkman KL, Minson JA, Volpp KGM. Holding the Hunger Games Hostage at the Gym: An Evaluation of Temptation Bundling. Manage Sci. 2014;60(2):283-299.

37. Otte-Trojel T, De Bont A, Rundall TG, Van De Klundert J. What do we know about developing patient portals? a systematic literature review. J Am Med Inform Assoc. 2015;23(e1):e162-e168.

38. Lyles CR, Nelson EC, Frampton S, Dykes PC, Cemballi AG, Sarkar U. Using Electronic Health Record Portals to Improve Patient Engagement: Research Priorities and Best Practices. Am Coll Phys. 2020;172:S123S129.

Publisher's Note: Springer Nature remains neutral with regard to jurisdictional claims in published maps and institutional affiliations. 